\title{
Elevator speech: Students' discussions of forces and acceleration by means of a scale in an elevator
}

\author{
Astrid Johansen and Berit Bungum \\ Department of Physics, Norwegian University of Science and Technology, Norway
}

Students' challenges in learning mechanics are well documented from test situations, and group discussions are considered a fruitful way to meet these challenges. In this paper, we present a study from an authentic teaching setting where upper secondary students in groups solve the task of calculating the acceleration of an elevator by means of a scale. The group work was audio recorded in three groups with different ability levels. Analysis was performed inductively using the analytical tool of semantic gravity and semantic waves. The results reveal multiple challenges solving the task, even among high-achieving secondary physics students. The study shows that for group discussions to be fruitful, students must be able to negotiate for meaning by alternating between different levels of semantic gravity. In this study, only the group consisting of relatively high-achieving students was able to do this. For the groups that did not succeed, this is found to be due to insufficient knowledge base, poor integration of the required concepts in their own language and inappropriate epistemological framing of the situation. It is concluded that more effort should be put into learning basic concepts than curricula and teaching traditions normally provide. This should include tasks carefully adapted to students' ability, where students can practice alternation between levels of semantic gravity.

\author{
ARTICLE DETAILS \\ LUMAT General Issue \\ Vol 10 No 1 (2022), 23-48 \\ Received 4 January 2021 \\ Accepted 8 December 2021 \\ Published 21 January 2022 \\ Pages: 26 \\ References: 30 \\ Correspondence: \\ astrid.johansen@ntnu.no \\ https://doi.org/10.31129/ \\ LUMAT.10.1.1475
}

Keywords: Mechanics, students' language use, group discussions, semantic waves

\section{Introduction}

Mechanics forms an essential part of the traditional content of school physics, but it presents students with major conceptual challenges. A range of studies have investigated students' misconceptions in mechanics over several (see e.g. Duit et al., 2014). The understanding of concepts is closely connected to how these concepts are integrated in our language (Brookes \& Etkina, 2009). In particular, the field of mechanics involves linguistic challenges, as 'force' and other concepts may have parallel everyday meanings (see e.g. Brookes \& Etkina, 2009; Halloun \& Hestenes, 1985; Williams, 1999).

The study reported in this article contributes to the field by investigating students' language use and reasoning and the challenges they encounter while working on a physics task in an everyday setting. The task involves finding the acceleration of an elevator by means of a bathing scale. This provides an opportunity to investigate the 
interplay between everyday talk and scientific physics discourse and how it affects students' reasoning.

The research questions are as follows:

1. What challenges do students encounter in discussing the problem of determining the acceleration by analyzing forces in an elevator?

2. What characterises students' discussions and strategies when solving problems in an everyday context in groups?

Recordings of student discussions in three groups working on the problem are analyzed in light of documented student conceptions in the research literature. A framework involving semantic gravity and semantic waves (Maton, 2013) is applied for analyzing characteristics of the student discussions and for identifying strategies the student groups use to solve the task. Together, the results provide a picture of students' challenges and reasoning in solving mechanics problems in an everyday setting.

\section{Theoretical perspectives}

\subsection{Student challenges in learning physics}

Research in physics education has provided substantial insight into the different types of challenges students face when learning physics. Within the topic of mechanics, a large number of research studies have been performed on students' misconceptions and struggle with concepts (e.g. Alonzo \& Steedle, 2009; Clement, 1982; Coelho, 2012), often with constructivism as a framework for learning and with the purpose of conceptual change (see Duit et al., 2014). Results show that students may be able to reproduce equations like $\mathrm{F}=\mathrm{m} \cdot \mathrm{a}$ (Newton's second law) without really understanding their conceptual content and that many students have mistaken ideas about the relationships between forces and motion. The key concept force is in fact very abstract and appears with diverse definitions and descriptions in physics teaching and textbooks (Alonzo \& Steedle, 2009; Stavrum et al., 2020; Touger, 1991). This makes it difficult for students to extract the concise meaning of the term (Itza-Ortiz et al., 2003; Williams, 1999). Brookes and Etkina (2009) argue that the concept of force is challenging due to a combination of linguistic and ontological difficulties. Students have experienced different meanings of force in different contexts, leading to ontological disambiguation, which in turn is often interpreted as misconceptions by 
researchers. In particular, the concept of weight carries different meanings (see Taibu et al., 2015); in everyday language, 'weight' may be synonymous to mass (e.g. "I have lost weight"). In Norwegian, an even more confusing element exists since the word 'weight' ('vekt') also describes the measuring devise (the scale). In order to support students' understanding of challenging scientific concepts, it is therefore recommended that teachers pay attention to ambiguous words and concepts that have different meanings in different contexts (Itza-Ortiz et al., 2003; Williams, 1999) and that students are given a variety of experiences with concepts over time (Bigozzi et al., 2014; Van Heuvelen, 1991). In line with this, many scholars have pointed to the importance of students using and developing their own language as part of the learning process and argued for the use of group discussions in teaching (Benckert et al., 2005; Heller \& Hollabaugh, 1992; Heller et al., 1992; Lemke, 1990; Mortimer \& Scott, 2003).

\subsection{Knowledge structures and activation}

Sabella and Redish (2007) describe the learning of physics as a result of building strong connections between several distinct clusters of concepts and principles, and the activation of one cluster will lead to activating of others, resulting in a pattern of association. This pattern of association may be described as the student's knowledge structures. The structures are the individual knowledge resources the student bases his or her thinking on and are not unambiguously right or wrong. For example, the knowledge resource "Force causes motion" can be correct in one context but wrong and representing a misconception in another. It has been shown that a student's knowledge organisation is a key aspect for distinguishing experts from novices (Chi et al., 1981). Knowledge structures may be activated by cues that students are explicitly or implicitly given in a task (Low \& Wilson, 2017). Cues may be specific keywords or features of a context, which activate specific knowledge structures. For novices, cues related to the specific context, such as the presence of an inclined plane or the word 'acceleration', often activate a specific memorised procedure, while experts use more abstract cues associated with physics principles (Chi et al., 1981). Cueing of knowledge structures can also be a part of the student's epistemological framing, meaning the student's judgement of which resources and strategies are most appropriate in a given context (Bing \& Redish, 2009; Redish, 2004). A frame is the individual's, often subconscious, answer to the question "What is it that's going on here?" and results in selective attention focused on deciding what is important in this particular case. The 
basis for this judgement is all prior experience and knowledge the student holds. This means that students might have very different epistemological framings of the same problem, and social and contextual conditions can play a subtle role as well. Scherr and Hammer (2009) emphasise the role of social framing, in which students form a sense of what to expect of each other, of their instructor and of themselves. For groups of students working together collaboratively, different aspects of framing interact and often change during different phases of the task (Scherr \& Hammer, 2009).

\subsection{Semantic gravity and semantic waves}

The process of learning physics is also about getting to know the culture and practices of physics. In physics, like every other knowledge domain, there are certain expert ways of performance. These 'rules of the game' are often tacit knowledge and known as legitimate codes (Lee \& Wan, 2020). Legitimate code theory (LCT) is a conceptional framework for exploring these codes. (Maton, 2013). LCT consist of multiple dimensions, each of them offers concepts for analyzing different organizing principles underlying practices. An important aim in learning physics is to organize knowledge in overarching principles reducing the dependency on the context of a problem (Bao \& Koenig, 2019). This can be analyzed using the concept from the dimension of Semantics in LCT: semantic gravity. Semantic gravity refers to the degree to which an expression is related to context, in other words, how specific and concrete it is. For example, when students discuss with each other while performing a physics experiment, utterances concerning what a person should do and what he or she will observe have strong semantic gravity. In contrast, utterances concerning the difference between Newton's first and third law at a general basis have weak semantic gravity.

An important feature of semantic gravity is that it forms a continuum from weak to strong (Maton, 2013). Maton dynamises this continuum to changes over time by bringing in the processes of weakening and strengthening the semantic gravity. This enables an analysis to include incidences of abstraction and generalisation from concrete situations, or conversely, transferring the general ideas to a concrete situation. Good competence in physics means the ability to alternate between levels, that is, to be able to weaken or strengthen semantic gravity. The semantic gravity can be presented as a function of time, resulting in a semantic wave (Maton, 2013). 


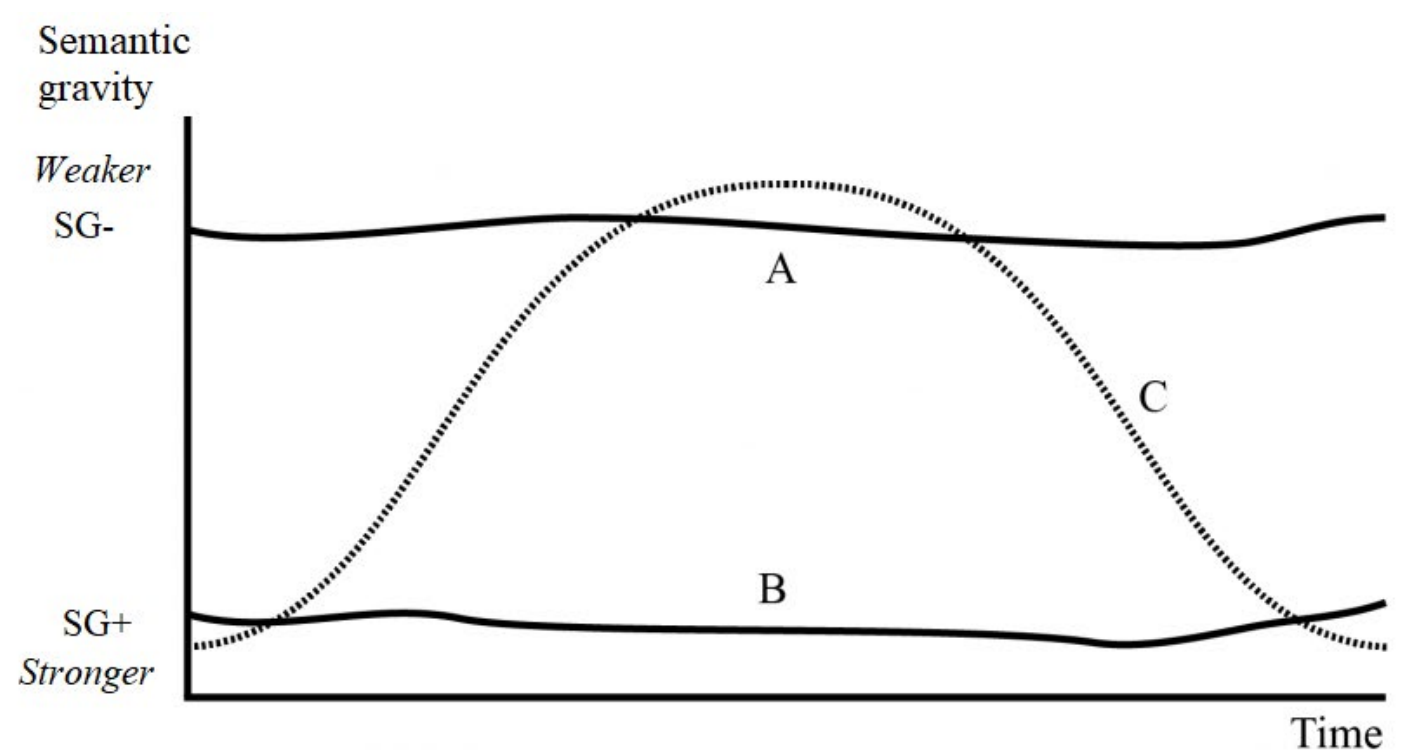

semantic

ranges

Figure 1. Different semantic profiles and their semantic range. "SG+" denotes strong semantic gravity and "SG-" denotes weak semantic gravity. (Based on Maton, 2013).

In Figure 1, profiles A and B describe situations where the variation in semantic gravity is low, leading to a small semantic range. In A, the talk stays at a general level, not bringing in anything anchoring it to context. In $\mathrm{B}$, the talk is always related to the specific context with no attempt to bring in general ideas. Neither of these two profiles supports new understanding (Maton, 2013). In cumulative knowledge-building, general principles have to be connected to actual situations. In other words, an alternation is required between different levels of semantic gravity (Georgiou et al., 2014; Maton, 2013). This is what we see happens in situation $\mathrm{C}$, where we have a semantic wave with a significant larger semantic range.

In this study, the concepts of semantic gravity and semantic waves are used to analyse how students' discussions contribute to solving a given physics problem.

\section{Teaching setting and research methods}

The study was undertaken in an upper secondary physics class where the tasks given to students formed part of their physics teaching. This authentic setting provides for insights on students' reasoning in a normal teaching situation rather than in a more artificial test setting. The research approach is a combination of meaning making based on condensation and interpretation of data to form a narrative and a more detailed and systematic analysis based on LCT. This is done in order to ensure both breadth and depth in the analysis. 
The data are from students in a Norwegian upper secondary physics class. Physics is an elective subject in Norwegian upper secondary schools, normally chosen by highperforming students who appreciate traditional subject-oriented teaching (Angell et al., 2004). The class in this investigation belongs to a school with a good reputation, and results and the teacher is well qualified.

\subsection{The physics problem to be solved}

The students were given the task of finding the acceleration of an elevator by doing measurements with a bathing scale. They had to figure out themselves how to solve this, but their investigation was guided by two questions given by the teacher:

- What force is measured by a bathing scale?

- What is the magnitude of the acceleration of the school's elevator?

The textbook used by the class in this study (Jerstad et al., 2013) included this problem in examples as well as exercises. However, since the task presented to students in this study involves everyday objects (a bathing scale and an elevator) and no obvious cues that pointed to a specific way of solving these problems, the link to the textbook was not clear for the students.

To solve the problem, the students need to analyse the relevant forces (see Figure 2) to realise that the acceleration is due to a varying normal force $(\mathrm{N})$ and that the force measured by the scale is the reaction force to this normal force $\left(\mathrm{N}^{*}\right)$. Further, they must realise that the net force is given by $\mathrm{N}-\mathrm{G}$ and apply Newton's second law to calculate the acceleration.

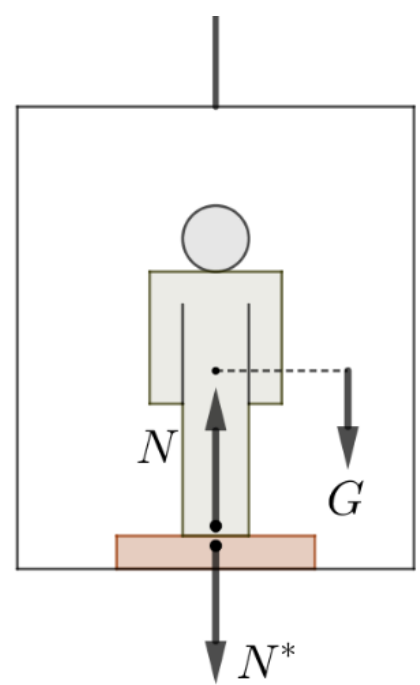

Figure 2. The relevant forces when the elevator accelerates upwards. 
Hence, the acceleration can be expressed as

$$
a=\frac{N^{*}}{m}-g
$$

The scale shows the result in 'kg' even though it represents a force, not a mass. Therefore, the students must translate the 'kg' shown on the scale into the correct unit, newton, by multiplying by $g=9,81 \mathrm{~m} / \mathrm{s}^{2}$.

\subsection{Teaching setting}

When the investigation was undertaken, the class had finished six weeks of instruction in mechanics, following the curriculum for introductory physics in upper secondary school in Norway. The students were to work in groups, and in order to allow discussion with peers on the same level, the class was divided into relatively homogenous groups of 3-4 students. This was done by the teacher based on his impression and the results of a test the students had taken prior to starting to work with mechanics. The students were to discuss what force the scale measures when a person stands on it and to find the acceleration of the elevator by performing measurements. After finishing the discussion, the student groups were equipped with an ordinary bathroom scale to bring with them into an elevator to implement the ideas they had developed during the discussion. The entire session lasted for 80 minutes.

The discussions in each of the three groups lasted for 25-35 minutes, with very few incidents of interruptions and talking outside the task. This indicates that students were motivated to solve the task. During the students' discussions and investigation, the teacher moved between the groups and guided the students towards a solution of the problem.

\subsection{Research methods}

We recorded discussions in three groups, where group 1 was considered as high achieving by the teacher, group 2 as rather low achieving and group 3 as intermediate. Thorough information was given to the students at the outset of the study, and all students participated voluntarily. The discussions were recorded by the students using mobile phones and sent to the teacher, who ensured anonymity in the titles of the audio files before he forwarded them to the authors. In this study, we focus on students' own reasoning and not on interaction with the teacher, and therefore only discussions where the teacher is not present are analysed. Further, we observed that 
the practical part of the task in the school's elevator led to the exchange of 'the correct solution' between groups, and therefore only discussions that occurred prior to the measurement in the elevator are included in the analysis. As a result, 446 utterances were coded for group 1, 182 for group 2 and 286 for group 3. The main reason for the variation in the number of utterances was a higher degree of teacher participation in the discussions in groups 2 and 3.

The recordings were fully transcribed verbatim. Non-verbal utterances, such as sighing and laughter, were indicated in cases where they could influence the interpretation of the words spoken. The analysis was undertaken in three stages. First, the focus was on the challenges the students met. To ensure all voices would be heard and all types of challenges would emerge, the transcripts were coded inductively, leading to the initial codes 'the normal force', 'force analysis', 'Newton's second law', 'confusing concepts and language', 'mathematics', 'use of equations of motion', 'connecting physics to experience' and the word 'WEIGHT'. These codes were categorised into three main areas of challenges: (i) the changing normal force, (ii) application of Newton's laws and (ii) language and everyday influence. The first category includes the challenge of understanding that the normal force varies and is responsible for the acceleration as well as the problems connected to the calculation of this force. The second category includes all problems combining the force analysis with the calculation of the acceleration. The third category includes challenges resulting from imprecise use of concepts and everyday language. The codes and areas were used for structuring the analysis and not in the presentation of results, where a more chronological approach is used.

To get a picture of how the discussions developed, utterances were also categorised based on their organising function. Utterances not related to the task were code as 'off-task', utterances concerning practical administration of the task (e.g. "who is writing?”) were coded as 'task organisation', abrupt thematic changes in the discussion were coded as 'thematic leaps' and utterances were students were talking over each other about different themes were coded as 'parallel discussions'.

Finally, to analyse how utterances contributed to progress in the discussion, the framework of semantic gravity and semantic waves (Maton, 2013) was applied. Utterances were coded based on their level of semantic gravity in three levels: strong (SG+), intermediate (IM), and weak (SG-). The code SG+ was given to utterances concerning the practical situation directly connected to the context with no link to general theory. 
An example of an utterance coded as $\mathrm{SG}+$ is

If a person is standing on a scale in an elevator moving upwards, then the weight will increase

Utterances coded as SG- dealt with general theory without connection to the actual situation, for example

The reaction force to the gravitation is the pull you exert on the earth

Utterances coded as IM included both strong and weak semantic gravity. An example is

So, when the elevator moves upwards, the force pushing upwards on the person increases

Here, general ideas about changing forces are applied to the concrete situation, thereby combining strong and weak levels of semantic gravity.

For utterances that contributed constructively to the previous utterance and at the same time changed the level of semantic gravity, the direction of the shift was coded with an arrow indicating weakening or reinforcement. For example, in a general discussion concerning Newton's second law, the utterance

If the mass is $10 \ldots$ and you suddenly have an acceleration of $1.5 \ldots$ then the sum of forces will increase

connects the general idea to something more concrete (numbers), resulting in a strengthening of semantic gravity, and it is therefore coded as SG- $\downarrow$. An example of weakening of semantic gravity occurs when students discuss why a bathing scale will show an increased weight when "moving upwards", and a student adds

By that ... we mean that the force pushing down on the scale will increase

Here, the concrete increase in ' $k g$ ' is related to the more abstract concept of 'force', resulting in a weakening semantic gravity. This utterance is therefore coded as SG+个. Based on changing semantic gravity, the discussions were mapped into semantic waves for the three groups. Details of this coding are presented in the Appendix.

\section{Results}

The analysis of the group discussions provides insights on both what challenges the students met and the strategies they used in their efforts to solve the problem. We first present the results regarding the students' challenges. Thereafter, we present an analysis of the discussions to reveal which strategies students used to solve the problem and how the dialogue contributed (or not) to progress in solving the task. 


\subsection{Challenges the students faced}

In order to allow all the groups to be heard, the results are not presented in terms of the codes used for analysis. Instead, we present the results structured within three themes across groups: 1) what force the scale measures, 2) the role of the normal force and 3) calculation of acceleration. These themes reflect the chronological development of the discussion in group 1. The discussions in group 2 and group 3 were more disorganised, but the same themes can be identified in these groups.

What force is measured by the scale?

The task given to students by the teacher begins with a question about what force the scale measures. In all three groups, the discussion starts with suggesting that the scale measures the gravitational force. Excerpt 1a from Group 1 shows that Vilma and Una are aware that the question is about the force on the scale, not on the person, but nonetheless they conclude that the force the scale shows is the gravitational force. Una describes it more correctly as "the size of the force you push against the scale" without discussing the distinction.

Excerpt 1a. "The size of the force you push down on the scale" (Group 1)

Vilma: Gravitation, I think

Synne: Yes

Vilma: The gravitation acts on it

Una: Yes, how ... in a way, the size of the force ... you push down on the scale

In group 2, the suggestion that the scale measures the gravitational force is challenged by students' knowledge of units. In Excerpt 1b, Sara and Rob argue that since the scale measures in $\mathrm{kg}$, it must be mass that is measured and not the gravitational force. At the end of the excerpt, Britt refers to the question given by the teacher ("What force is measured by the scale?") to argue that the answer is in terms of force and not mass. The students do not seem to see the connection for the scale even though Sara has stated that you find the force (weight) by multiplying mass by the acceleration due to gravity, $g$. Based on their utterances, they do not seem to realise that the transfer from force to mass is inherent in the way the scale measures force.

Excerpt 1b. "The scale measures mass, not force" (Group 2)

Britt: Which force it measures? Isn't it the gravitational force? 
Sara: No ...

Rob: It doesn't measure in newton

Britt: Well, it does measure

Sara: It measures in kilos

Rob: ... in kilos, yes

Britt: But when a person is standing on the scale, it will measure how much pressure you push down on it ... or how much your mass pushes down on the scale

Sara: Yes?

Britt: ... and that is what the gravitational force does

Sara: Sort of. But if you calculate the weight of a person, you multiply the mass with the acceleration due to gravity. But the scale measures the mass of a person.

Britt: Yes, but the task says «what force» not what it measures

In group 3, the discussion starts with Jan asserting that what the scale measures must have to do with gravitation since the scale would show a different value on the moon (see Excerpt 1c). In an attempt to analyse forces, Allan conduses the reaction force with a balancing force in stating that the scale being at rest makes the force and reaction force equal. Jan, on the other hand, describes the force measured by the scale as the gravitational force on the object on the scale, failing to consider what object the force in question works on.

Excerpt 1c. "The scale measures gravitational force" (Group 3)

Jan: It measures how much the gravitational force affects the thing placed on the scale ... in a way? ... Because the scale had shown differently ... for instance on the Moon. So it has something to do with the gravitational force.

\section{(...)}

Allan: The scale is at rest, and this decides whether the force and the reaction force have the same size. When these two forces have the same size, it means that the scale is at rest. Which it is.

Eva: So which force do you mean is measured by the scale?

Jan: The influence of the gravitational force on the object on the scale ... I believe. It measures the weight of an object.

The role of the normal force

Excerpt 2a shows how Synne in group 1 brings the normal force into consideration in discussing the situation when the elevator is moving downwards. The students use everyday experience, which tells them that you will not fall through the elevator floor when it is moving or accelerating. There must be a force - the normal force - that 
"keeps you up". Synne explains it as "a little bit of force" that pushes her upwards or keeps her where she is. This indicates that Synne sees the need for a force that counteracts the gravitational force, and she expresses that the normal force does not have to be as big when the elevator is going downwards as it is when going upwards. This is a constructive move towards analysing the situation with Newton's second law. However, it does not seem that Synne is able to distinguish between acceleration and movement, and that she does not consider forces on the same object. Rather, she compares the normal force from the scale on herself to the force she pushes down on the scale, but without identifying these as action and reaction forces and hence identical in magnitude according to Newton's third law.

Excerpt 2a. "A little bit of force pushes me upwards" (Group 1)

Synne: I'm going down ... but this normal force won't be as big ... will it?

Una: But you still won't fall through the floor?

Synne: No ...

Una: It is still this force that keeps you up?

Synne: Yes, ... so it is a little bit of force that pushes me upwards ... or keeps me where I am?

Una: Yes ...

Synne: But when the elevator is going down, it will ... The normal force doesn't need to be as big ... It doesn't need to be as big as the force I push down with, in a way.

Although the discussion in group 1 is heading towards a correct description of forces and interaction, there is still confusion in the group. Questions that came up after the sequence shown in Excerpt 2a were, for example, whether "the gravitational force is larger when the elevator is moving upwards" and "why the weight is heavier when the elevator is moving upwards". Despite this confusion, the group manages to reach a solution for how forces act in the elevator using Newton's $3^{\text {rd }}$ law to correctly explain that the force on the scale is the reaction force to the normal force, that is, the force $N^{*}$ in Figure 1.

In group 2, Sara makes the correct statement that the scale measures the reaction force to the normal force, shown in Excerpt 2b. However, they get off track because they feel a need to calculate the normal force since the scale measures its reaction force. The further discussion then leads to two misconceptions that prevent progress: first, that the normal force $N$ equals the weight $G$ in magnitude, and second, that this is not the case since $N$ and $G$ are not reaction forces. It seems from Excerpt $2 b$ that 
the students jointly refer to situations they have worked with where the normal force equals the gravitation force when there is no acceleration. They seem to mix up Newton's third law with Newton's second law with no acceleration, where the normal force will be a balancing force to the gravitational force.

Excerpt 2b. "The reaction force to the normal force" (Group 2)

Sara: Well, I think it is the reaction force to the normal force that it calculates, so to say.

Sara: But what was the way to calculate the normal force again?

Britt: The normal force is equal to the gravitational force.

Sara: No, because they are not reaction forces.

Britt: No, no, but they usually are.

Sara: Yes, I understand what you... yes.

Britt: At least when you stand on... when you are not moving.

In group 3, Eva brings in the normal force when analysing forces on the person on the scale, as shown in Excerpt 2c. She considers the normal force to be smaller than the gravitational force, apparently because she believes that a net force is required in order for the scale to show a weight. This indicates confusion about which object is considered. Allan disagrees by referring to the fact that the scale is initially at rest, but again he mixes up the reaction force with a balancing force, and in addition "at rest" is used rather than zero acceleration. At the end of Excerpt 2c, Allan shows a growing understanding of what force the scale measures, which he calls "the reaction force to the force that acts from the body and up". However, since he uses the term "reaction force" to refer to a balancing normal force, this becomes "the reaction force to the reaction force".

Excerpt 2c. "The reaction force to the reaction force" (Group 3)

Eva: If you place yourself on a scale ... you'll push it down ... but there is also something that is pushed upwards ... and this must be the normal force. Right? (...)

Eva: Then the normal force has to be smaller than the gravitational force, because you still are pushed down ... because the weight is still downwards

Allan: No, I wouldn't say so. Because the scale is at rest, and this decides whether the force and the reaction force have the same size. When these two forces are equal, it means that the scale is at rest. Which it is.

(...) 
Allan: ... but the scale measures ... it will vary. So, if the scale measures the reaction force ... No (interrupts himself) ... the reaction force to the reaction force, if you see what I mean? The reaction force to the force acting from the object and up.

\section{Calculating the acceleration}

In the phase of performing measurement and calculations, the students in group 1 consider how acceleration can be calculated during the elevator ride. In Excerpt 3a, Synne suggests comparing the weight the scale shows at rest with what it shows when the elevator is moving. The students seem to agree that the difference in what the scale shows is related to acceleration, and Synne appears to be looking for some sort of ratio. Vilma's response "Which formula is to be used?" indicates a belief that physics is about choosing the correct formula to solve a problem.

\section{Excerpt 3a. "Which formula is to be used?" (Group 1)}

Synne: In a way, you must have ... a starting point, with ... the weight you actually have on the scale ... when it doesn't move. And then you have to compare to what it says when you ... when the elevator moves.

Vilma:Yes. But I don't understand how we shall ... How do you calculate the acceleration ... compared to ... Which formula is to be used?

Following the sequence in Excerpt 3a, the students conclude that the formula to use is Newton's second law: force equals mass times acceleration. New challenges arise when the students attempt to use the measured values on the scale to calculate the elevator's acceleration. In Excerpt 3b, they are back to the confusion about what weight means, and they do not seem to be able to use the analysis of forces they already conducted or to consider the normal force.

Excerpt 3b. "We don't know the size of the force" (Group 1)

Una: What? ... But how can we ...? We know the weight, because we can see it on the scale

Synne: Yes

Vilma: Yes

Una: But we don't know the force ... and we don't know the sum of the forces ... and we don't know the acceleration

Una: Yes, but ... how big is the force pushing upwards? That's what we need to know! It is ...

Vilma: Isn't it just to take your weight and ...?

Una: But we don't know the size of the force the elevator pushes upwards on us? 
The solution becomes possible in group 1 when the students realise that 'weight' becomes a force when the mass is multiplied by $g$, as shown in Excerpt 3c. Here, Synne brings in the key point that the force representing what is measured on the scale equals the force executed by the person standing on it and that this enables them to calculate the acceleration from Newton's second law.

\section{Excerpt 3c. "The force pushing downward from us" (Group 1)}

Synne: Yes, we get the sum of the forces ... because when we have the number in $\mathrm{kg}$ from the scale ... And then we multiply by $\mathrm{g} . .$.

Vilma: yes ...

Synne: ... then we have the sum of the forces ... And then we can take ... eh, the sum of the forces divided with $\mathrm{m}$. Then we will get a.

Vilma: mhm

Una: Then we have to take ... that weight ... the weight the scale shows ... when the elevator moves up ... times g, $9,81 \mathrm{~m} / \mathrm{s}^{2}$

Synne: Yes! Then we will get the sum of the forces

Una: No, then we will get the force pushing downwards from us.

In Group 3, the students approach the problem of calculating acceleration by formulating a strategy of comparing two situations and analysing the difference in the weight measured on the scale (see Excerpt 3d). Here, Jan states that the scale will show a larger weight when the elevator moves upwards because an upwards force is acting. This could have been a constructive way forward, but Newton's second law is never brought into the discussion. Instead, the group proceeds without attempting to solve the problem by considering the equations of motion they have learnt earlier, and they never succeed in solving the problem.

\section{Excerpt 3d. "Weight increases by an upwards force" (Group 3)}

Jan: We have to measure something on the scale before we enter the elevator, and then find the weight. Afterwards we do the same inside the elevator [being interrupted by Allan]

Allan: A person has to stand in the elevator to read the value

Jan: Yes, on the scale while it's moving upwards

Jan: ... and then it weighs more, because it is a force upwards. It seems logical.

Allan: Yes

Jan: And the difference ... The weight will be higher ... The difference in mass is due to that the acceleration is higher ... right?

Allan: Yes ... 
Jan: ... because the elevator is moving upwards

Allan: What I think is, when we are going ... when we are entering the elevator, we have to place the scale on the floor. Then we put a weight on it. And when it moves upwards ... when the elevator moves upwards, the mass will... or the mass ... or the weight ... the elevator shows bigger...

A parallel situation to this takes place in group 2, where Sara describes the force on the scale correctly as the reaction force to the normal force, but she has no constructive strategy for using this to calculate the acceleration based on Newton's laws (Excerpt 2b). It seems that the students can manage to analyse forces when asked to do so directly but have major problems applying the results of this analysis.

\subsection{Detailed analysis of student discussions}

In this section, we analyse how the groups are negotiating for meaning in the discussions and which strategies they use in their effort to solve the task.

Organising structure of the discussions

To give an overall picture of how the discussions took place, the results of the analysis of organising characteristics of the discussions are shown in Table 1.

Table 1. Organising characteristics of the discussions

\begin{tabular}{lccccc} 
Group & \# Utterances & \# Off task & $\begin{array}{l}\text { \# Task } \\
\text { organisation }\end{array}$ & $\begin{array}{c}\text { \# Thematic } \\
\text { leap }\end{array}$ & $\begin{array}{c}\text { \# Parallel } \\
\text { discussions }\end{array}$ \\
\hline 1. Una, Synne, Vilma & 446 & 4 & 7 & 4 & 0 \\
2. Sara, Rob, Britt & 182 & 0 & 21 & 3 & 6 \\
3. Allan, Eva, Jan & 286 & 10 & 49 & 13 & 19 \\
\hline
\end{tabular}

The results show that all three groups had a low number of utterances not related to the task, but we see much more attention paid to task organisation in groups 2 and 3. In addition, group 3 stands out negatively concerning flow and joint participation in the discussion.

Content and progress of the discussions

Here, we present semantic waves for the three groups illustrating how the discussions proceed. The level of semantic gravity is presented as a function of the utterance number, which in turn represents time. The results of this analysis are shown in Figures 3, 4 and 5 for the part in the discussion where the groups discuss why the 
reading on the scale changes when the elevator moves upwards. The coded transcripts are presented in the Appendix.

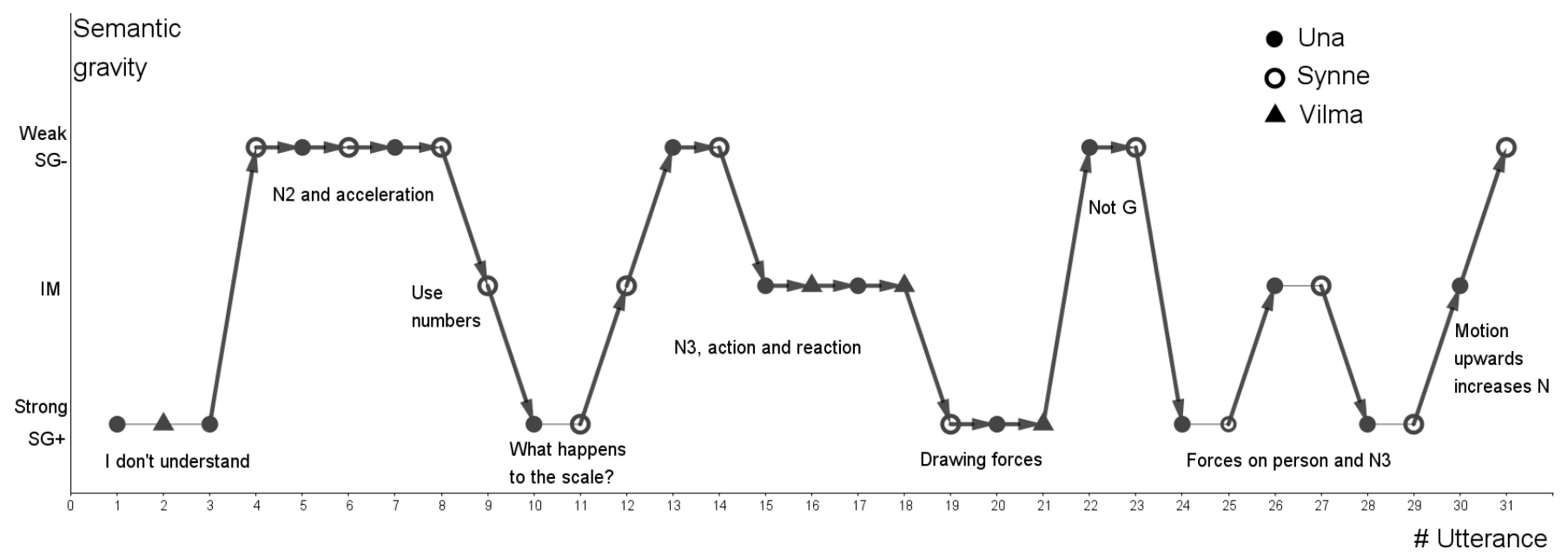

Figure 3. Semantic wave for group 1. Utterances actively following and contributing to the preceding utterance are marked with a bold arrow. Subsequent utterances that are solely confirmative or touching on the same topic without internal connection are marked with a thin line. Utterances with no connection to the preceding utterance are unconnected in the illustrations. In addition, the contribution of each student is shown by a specific sign, and key words for the discussion content are added.

In the first utterances in the discussion in group 1, the concrete situation is the topic when the students are struggling to find a starting point. Then, general principles, Newton's second law and acceleration, are brought in, but with no connection to the actual situation concerning the scale. This leads to an abrupt transition to weak semantic gravity. The idea of using numbers makes the situation less general and increases the level of semantic gravity. The discussion is then brought even closer to context by bringing in the scale. Newton's third law is included related to the scale, and further discussion concerning Newton's third law alternates between the weak and intermediate level. Una has understood an important point in utterance \# 17, but Vilma is not following. Synne responds by distilling it all down to the concrete situation by saying, "Let's draw, folks!" The discussion continues at a strong level of semantic gravity, but more general ideas about gravitational force and reaction forces are mentioned, mostly (it seems) to ensure they are on the right track. In the last part of the discussion Una gives an almost complete explanation before the teacher stops by, interrupting the discussion. 
From Figure 3, we see that all the utterances in the discussion in group 1 are connected. This indicates a continuous and coherent discussion. We see that most of the utterances are contributing to the preceding one, indicating that the students are listening and responding to each other. We find many alternations between different levels of semantic gravity, and they actively use the intermediate level in these alternations to connect the actual situation to general physical principles. Una and Synne are the most active participants in the discussion, but Vilma makes important contributions to their common meaning-making by posing questions and asking for explanations.

In addition, the analysis of semantic gravity reveals some of the strategies used by group 1. We find that they activate relevant general theoretical principles and connect this to the actual situation by giving values for physical quantities. They look for consequences of theory in the actual situation, and they connect real-life observations to general physical theory. By drawing a force diagram, they manage to utilise a different representation. At the end, they are evaluating their ideas based on relevance and checking for consequences of their idea if the circumstances are changing. Together, this constitutes a toolbox that enables cumulative learning and the development of understanding.

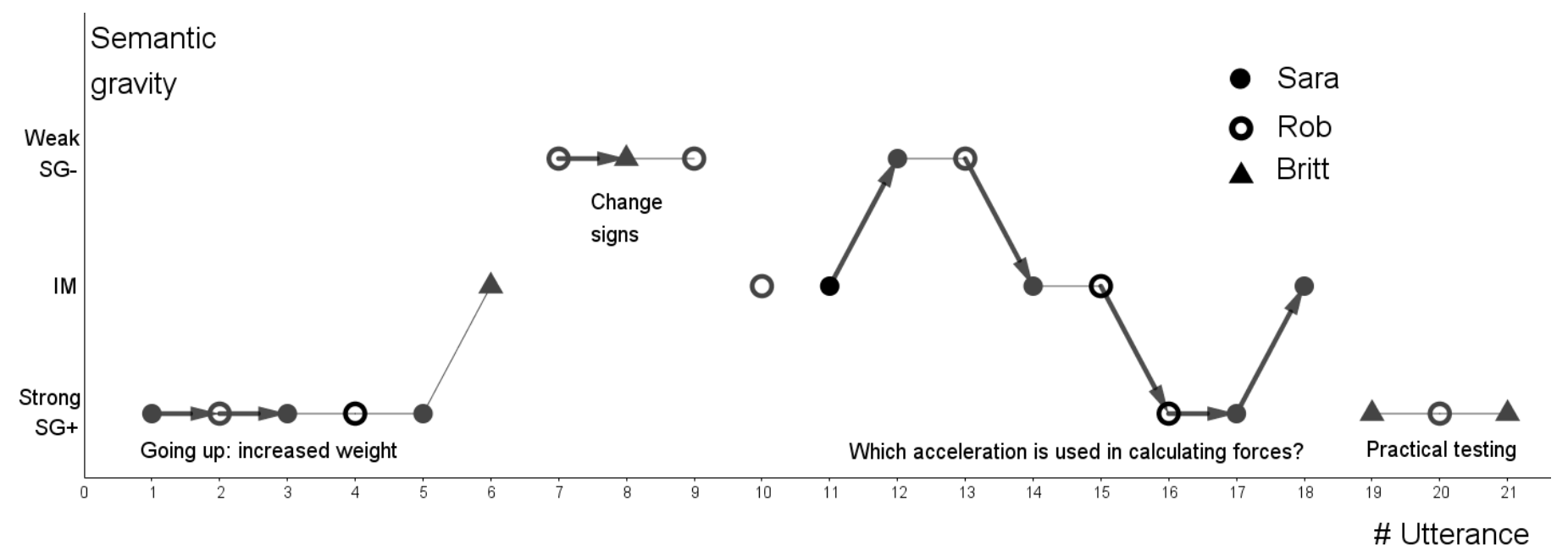

Figure 4. Semantic wave for group 2. See Figure 3 for explanations.

The semantic wave of group 2 is shown in Figure 4. Here, the discussion starts at a strong level of semantic gravity dealing with their hypothesis that the scale will show an increased weight when moving upwards. To search for an explanation, Britt 
mentions the gravity force and repeats that there is motion, lifting the utterance to the intermediate level but without relating it to the previous utterance. Rob brings in the choice of positive direction at a general level, and this is discussed unconnected to the situation in the elevator. Sara starts her own reasoning concerning how to calculate the forces when the elevator is in motion opposed to when it is standing still. Even though Rob is supporting her with confirmative utterances, Sara is the only one contributing to this reasoning. She tries to connect Newton's laws to the actual situation but is not able to come to a meaningful conclusion. To progress in the work, Britt strengthens the semantic gravity of the discussion by suggesting they do the practical experiment, and this is discussed in the final utterances.

From Figure 4, we see that the discussion in group 2 includes breaks and unconnected utterances. Although we find several utterances contributing to the preceding one, this is mostly due to Sara following her own reasoning. Even though the discussion moves between all three levels of semantic gravity, the students are not able to utilise this change in abstraction level to make progress in their reasoning. The discussion is partly connected, with a different student being active in each part.

In this discussion, we can observe from the analysis that the strategies the students in group 3 are using consist of anchoring the problem in the actual context by discussing what will happen to the scale, bringing in general principles which might influence the situation, searching for formulas and postponing challenges. Together, these are not sufficient or effective strategies for students to be able to use the discussion to develop new meanings and understandings.

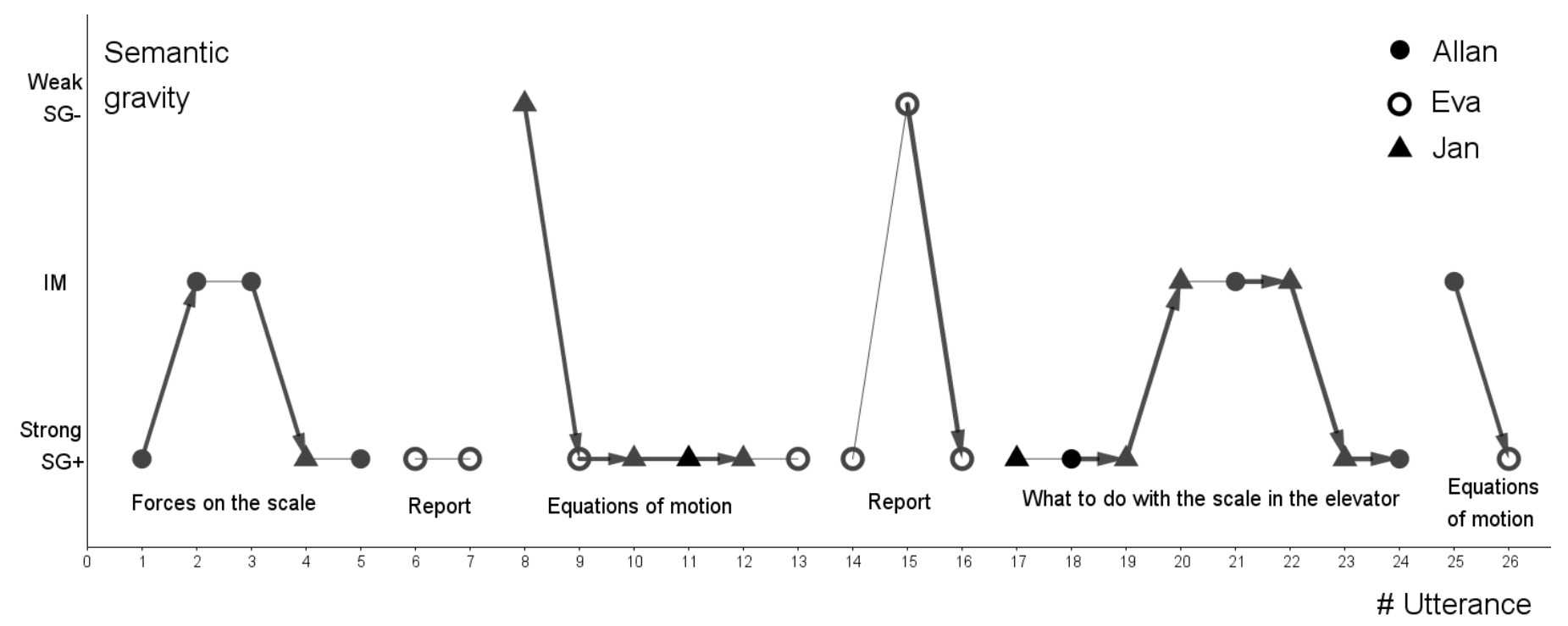

Figure 5. Semantic wave for group 3. See Figure 3 for explanations. 
The semantic wave of group 3 is shown in Figure 5. During the first utterances, the discussion alternates between strong and intermediate levels of semantic gravity when Allan discusses the forces acting on the scale and comes to a correct conclusion. Eva does not participate in the discussion but is concerned with how the report should be written. There is no response to this. Instead of following Allan's ideas concerning forces, Jan introduces the general idea of equations of motion and lifts the discussion to weak semantic gravity. In the following utterances, this is discussed at a strong level of semantic gravity dealing with practical measurements in the elevator. Eva continues her attention to the report-writing, mentioning Newton's second law at a general level, while Jan and Allan continue at a strong level of semantic gravity, thinking about the practical measurements in the elevator. Jan and Allan agree that the scale will show an increased weight when the elevator moves upwards and lift the discussion to the intermediate level by connecting the increase to an increase in acceleration. Even though their thinking is on the right track, Allan leaves the forces and brings in the equation of motion. The last two utterances deal with how the equation could be used.

In Figure 5, we see a fragmented discussion with distinct breaks between topics. Further, we can observe that several topics are addressed simultaneously, which might indicate that each student is focused on his or her own ideas rather than being sensitive to others. Most of the discussion deals with topics closely connected to the context, which is expressed as strong semantic gravity in the figure. There are incidences of utterances with weak semantic gravity, but the group is not able to take advantage of the intermediate level to make a connection between abstract theory and the concrete context. In this discussion, the attention is on how things should be done. There are few signs of effort to understand and explain connections.

The strategies we can observe in group 3 consist of anchoring the problem in the actual context by discussing what will happen to the scale, task organisation and searching for formulas. These strategies are not sufficient and partly inappropriate to develop meaning and understanding.

\section{Discussion}

This study has investigated the work of three student groups on the task of finding the acceleration of an elevator by means of a scale. It represents a case study, and no generalisations can be made. We have also given our own interpretations of the students' understandings in the presentation of results, and other interpretations 
might be possible. Despite these limitations, the study shows that students face a diversity of interrelated challenges in solving the task. Even for high-performing students, it is striking how difficult the task becomes, considering that they have been taught all the necessary tools to solve it. It is noteworthy, however, that the problems students encounter in solving the task are not primarily due to typical misconceptions or an Aristotelian view of dynamics as described in research on student conceptions in physics (e.g. Duit et al., 2014). Rather, the problems that emerge through the student discussions relate to identifying the forces that cause acceleration, combining and using Newton's laws correctly and distinguishing between Newton's second and third law. The students also show problems in keeping track of which system or object is considered, and they tend to mix up the reaction force with a balancing force when there is no acceleration. In accordance with earlier findings in physics education research (see Taibu et al., 2015), the concept of weight is also challenging for students. Some of the students seem to interpret weight as an intrinsic property of a body just like mass, probably influenced by everyday language and in line with how force can be interpreted as a property (Stavrum et al., 2020). The conceptual challenges are magnified by the fact that the force in question, $\mathrm{N}^{*}$, does not have a specific name except "the reaction force to the normal force". This makes it hard for students to grasp the overall logic of the problem. The students also have problems translating the 'kg' the scale shows to a force measured in newton, whereas they probably would have had no problems with a task described as "Find the gravitational force on an object with mass m".

One aspect of the task that makes it challenging is that it does not give students the typical cues that can activate the relevant knowledge structures to guide them to specific procedures for solving the task, as described by Low and Wilson (2017). Rather, it seems that the occurrence of the forces $\mathrm{N}$ and $\mathrm{G}$ prompts a mistaken strategy to equate the two, and in group 3 the term 'acceleration' might have been a cue to make use of the equations of motion rather than force analysis. This is in line with how Sabella and Redish (2007) have shown that cues may hinder rather than support students' activation of their knowledge structures. In addition, the students' and teacher's epistemological framings (Redish, 2004) seemed to play a role in dealing with the question "What force is measured by a bathing scale?" The students interpreted the question in an everyday context, where the answer is obviously "the weight", which they translated into gravity force in order to relate it to the physics context. The teacher, on the other hand, asked the question in an attempt to guide the 
students' reasoning towards an analysis of forces and, in this way, tried to connect general principles to context. None of the groups followed this guidance, which may be due to the different epistemological framing between the teacher and students.

Further, it seems that the everyday setting makes the task more challenging rather than easier. In particular, the discussions indicate that students have no everyday conception of the normal force, resulting in challenges in attempting to analyse the situation with everyday equipment, even though the students have analysed forces correctly from a physics point of view. It is interesting to note that all three groups use the word 'moving' rather than acceleration in the context of the elevator. In contrast, they include the word 'acceleration' when the discussion involves Newton's second law, showing that acceleration has a place in their way of talking about physics but does not form part of their epistemological framing of the situation in an everyday setting. It seems that this does not cause problems in the qualitative discussions of the physics problem, which may signify that they have similar epistemological frames and thus shared interpretations.

Although the task was a struggle for all of the students, it is clear from the results that the students in group 1, judged by the teacher as high achieving, have significantly better resources in terms of knowledge elements, the links between them and strategies for using them. In agreement with the teacher's more intuitive judgement of ability, this group is able to link generalised physics theory to the specific situation with the scale in the elevator. The semantic waves resulting from the analysis show that group 1 is able to switch between different levels of semantic gravity in a way that the other two groups are not capable of.

We also find from the results that while the everyday concept 'weight' leads to confusion in groups 2 and 3, it stimulates new questions in group 1, for example, in Excerpt $3 \mathrm{~b}$ where they distinguish between weight (meaning the mass that they read on the scale) and the force and what objects forces are acting on. This seems to be due to a better understanding of the basic concepts and principles in mechanics in this group than in the other two groups.

The students' epistemological framing is important for their choice of strategies (Bing \& Redish, 2009; Redish, 2004; Scherr \& Hammer, 2009). The parts of the discussions analysed by means of semantic gravity reveal that some of the lowerachieving students in groups 2 and 3 perceive the task as a practical task, activating a frame related to how a practical experiment should be carried out. This leads to focusing on equipment, measurement and writing a report. Another example of an 
activation of an epistemological framing that is not appropriate for solving the task is found in group 3. Here, the task "find the acceleration" seems to cue for the use of the equations of motion, not Newton's second law. In this case, the epistemological frame for calculating quantities connected to motion seems to dominate the one connected to the use of Newton's laws. One might speculate that this is due to students favoring strategies involving a standard procedure that only requires the use of the correct formula, compared to use of Newton's laws, which requires a higher level of understanding. Different epistemological framings may also have influenced how the students perceive the role of the discussion. The students in group 1 appear to use the discussion as intended: as a tool to develop a common understanding. In contrast, the students in groups 2 and 3 seem to perceive the discussion as a tool for deciding what should be done, concerning, for example, "how shall we measure?" or "what shall we write in the report?"

Despite all the challenges students face, the task about the scale in the elevator has good potential for building an in-depth understanding of mechanics. Through the discussion, students encounter multiple issues that must be resolved, and the new questions generated by the discussion may contribute to students' learning. The task provides good opportunities to relate abstract physics knowledge to everyday situations and to practice the alteration between levels of semantic gravity. However, in order to realise this potential for learning, the students must have access to the concepts needed to discuss the problem. Therefore, it is important that the teacher carefully considers progression, the use of different contexts and angles and sufficient time to allow the students to establish a coherent conceptual framework (Bigozzi et al., 2014; Sabella \& Redish, 2007; Van Heuvelen, 1991). Another important factor for the teacher to consider is raising awareness among the students of the purpose of the task and the role of the discussion. According to Bing and Redish (2009), awareness of different epistemological framings can reveal failures in communication between the teacher and students as well as between students. They argue that teachers need to be more explicit regarding which epistemological framing the task requires.

Our study has revealed a multiplicity of challenges students encounter when attempting to solve a physics task in an everyday setting. The bathroom scale, showing the force in 'kg', causes a lot of 'noise', which seems to be quite disturbing to students in their efforts to understand the physical relationships. We have also shown that only high-ability students are able to alter between levels of semantic gravity as required for solving the task. We therefore suggest exchanging the bathroom scale with a force 
platform (showing the force in newton) in order to avoid the confusion about the concept of weight. Alternatively, the force analysis might be performed with a force sensor lifting an object. In this way the normal force is omitted, and the task might be more straightforward to solve.

Regardless of how the task is presented, it is essential that this learning activity is a part of well-planned, long-term teaching in order for concepts to be established and strategies to be learned. Students might be asked to discuss and make predictions about forces in a variety of specified situations, in line with the recommendations of Bigozzi et al. (2014) about varied experiences with concepts over time. If this is followed by experiments in the elevator, measuring forces with a datalogger, the predictions can be compared to the graph from a datalogger providing rich opportunities for discussions at different levels. By carefully choosing parts of the graph corresponding to different levels of complexity, students might be able to connect the actual situation to the general ideas. This might give a wider range of students the opportunity to utilise discussions for learning physics.

\section{Conclusion}

The results of the study illustrate that analysing forces and applying the result in own reasoning and calculations are demanding for students. Based on the students' discussions of the task of analysing forces in an elevator, we find that the challenges they face are due to a weak knowledge base, problems integrating physical concepts in their natural language and improper epistemological framing for the task.

The study shows that for group discussions to be fruitful, leading to meaning and understanding for each individual student, alternations between different levels of semantic gravity are required. These alternations ensure connection between the actual problem and general theory. In the problem discussed in this study, only the group of students characterised as high achieving by the teacher was able to perform this alteration in solving the problem given. In the other two groups, the students did not have a sufficient knowledge base, they had not integrated the required concepts in their own language and their epistemological framing of the situation was not appropriate.

This indicates that assimilation of physics concepts into students' knowledge structures is more demanding than curricula and teaching have traditionally taken into account. Based on the results of the present study, we will argue that there is a need to spend more time and effort on basic concepts in the physics classroom, and 
this should include tasks carefully adapted to students' ability, where students can practice alternation between levels of semantic gravity.

\section{References}

Alonzo, A. C., \& Steedle, J. T. (2009). Developing and assessing a force and motion learning progression. Science Education, 93(3), 389-421. https://doi.org/10.1002/sce.20303

Angell, C., Guttersrud, Ø., Henriksen, E. K., \& Isnes, A. (2004). Physics: Frightful, but fun. Pupils' and teachers' views of physics and physics teaching. Science Education, 88(5), 683-706. https://doi.org/10.1002/sce.10141

Bao, L., \& Koenig, K. (2019). Physics education research for 21st century learning. Disciplinary and Interdisciplinary Science Education Research, 1(1), 2. https://doi.org/10.1186/s43031019-0007-8

Benckert, S., Petterson, S., Aasa, S., Johansson, O., \& Norman, R. (2005). Gruppdiskussioner rundt kontextrika problem i fysik - Hur ska priblemen utformas? Nordina, 1(2), 36-50. https://doi.org/10.5617/nordina.481

Bigozzi, L., Tarchi, C., Falsini, P., \& Fiorentini, C. (2014). 'Slow Science': Building scientific concepts in physics in high school. International Journal of Science Education, 36(13), 2221-2242. https://doi.org/10.1080/09500693.2014.919425

Bing, T. J., \& Redish, E. F. (2009). Analyzing problem solving using math in physics:

Epistemological framing via warrants. Physical Review Special Topics - Physics Education Research, 5(2), 020108. https://doi.org/10.1103/PhysRevSTPER.5.020108

Brookes, D. T., \& Etkina, E. (2009). "Force," ontology, and language. Physical Review Special Topics - Physics Education Research, 5(1), 010110. https://doi.org/10.1103/PhysRevSTPER.5.010110

Chi, M. T. H., Feltovich, P. J., \& Glaser, R. (1981). Categorization and representation of physics problems by experts and novices. Cognitive Science, 5(2), 121-152.

http://www.sciencedirect.com/science/article/pii/So364021381800298

Clement, J. (1982). Students' preconceptions in introductory mechanics. American Journal of Physics, 5o(1), 66-71. https://doi.org/10.1119/1.12989

Coelho, R. L. (2012). Conceptual Problems in the Foundations of Mechanics. Science \& Education, 21(9), 1337-1356. https://doi.org/10.1007/s11191-010-9336-X

Duit, R., Schecker, H., Høttecke, D., \& Niedderer, H. (2014). Teaching Physics. In N. G. Lederman \& S. K. Abell (Eds.), Handbook of Research on Science (Vol. 2, pp. 434-456). Routledge.

Georgiou, H., Maton, K., \& Sharma, M. (2014). Recovering Knowledge for Science Education Research: Exploring the "Icarus Effect" in Student Work. Canadian Journal of Science, Mathematics and Technology Education, 14(3), 252-268. https://doi.org/10.1080/14926156.2014.935526

Halloun, I. A., \& Hestenes, D. (1985). Common sense concepts about motion. American Journal of Physics, 53(11), 1056-1065. https://doi.org/10.1119/1.14031

Heller, P., \& Hollabaugh, M. (1992). Teaching problem solving through cooperative grouping. Part 2: Designing problems and structuring groups. American Journal of Physics, 6o(7), 637644. https://doi.org/10.1119/1.17118

Heller, P., Keith, R., \& Anderson, S. (1992). Teaching problem solving through cooperative grouping. Part 1: Group versus individual problem solving. American Journal of Physics, 6o(7), 627-636. https://doi.org/10.1119/1.17117 
Itza-Ortiz, S. F., Rebello, N. S., Zollman, D. A., \& Rodriguez-Achach, M. (2003). The Vocabulary of Introductory Physics and Its Implications for Learning Physics. The Physics Teacher, 41(6), 330-336. https://doi.org/10.1119/1.1607802

Jerstad, P., Sletbak, B., Grimenes, A. A., Renstrøm, R., Holm, O. B., \& Nymo, M. (2013). RomStoffTid 1 - Fysikk 1. Cappelen Damm.

Lee, Y.-J., \& Wan, D. (2020). How Complex or Abstract Are Science Learning Outcomes? A Novel Coding Scheme Based on Semantic Density and Gravity. Research in Science Education. https://doi.org/10.1007/s11165-020-09955-5

Lemke, J. L. (1990). Talking Science: Language, learning, and values. Ablex

Low, D., \& Wilson, K. (2017). The role of competing knowledge structures in undermining learning: Newton's second and third laws. American Journal of Physics, 85(1), 54-65. https://doi.org/10.1119/1.4972041

Maton, K. (2013). Making semantic waves: A key to cumulative knowledge-building. Linguistics and Education, 24(1), 8-22. https://doi.org/https://doi.org/10.1016/j.linged.2012.11.005

Mortimer, E., \& Scott, P. (2003). Meaning making in secondary classrooms. Open University Press.

Redish, E. (2004). A Theoretical Framework for Physics Education Research: Modeling student thinking. arXiv: Physics Education.

Sabella, M. S., \& Redish, E. F. (2007). Knowledge organization and activation in physics problem solving. American Journal of Physics, 75(11), 1017-1029.

https://doi.org/10.1119/1.2746359

Scherr, R. E., \& Hammer, D. (2009). Student Behavior and Epistemological Framing: Examples from Collaborative Active-Learning Activities in Physics. Cognition and Instruction, 27(2), 147-174. https://doi.org/10.1080/07370000902797379

Stavrum, L. R., Bungum, B., \& Persson, J. R. (2020). "Never at rest": developing a conceptual framework for descriptions of 'force' in physics textbooks. Nordina, 16(2), 16.

Taibu, R., Rudge, D., \& Schuster, D. (2015). Textbook presentations of weight: Conceptual difficulties and language ambiguities. Physical Review Special Topics - Physics Education Research, 11(1), 010117. https://doi.org/10.1103/PhysRevSTPER.11.010117

Touger, J. S. (1991). When words fail us. The Physics Teacher, 29(2), 90-95. https://doi.org/10.1119/1.2343227

Van Heuvelen, A. (1991). Learning to think like a physicist: A review of research-based instructional strategies. American Journal of Physics, 59(10), 891-897. https://doi.org/10.1119/1.16667

Williams, H. T. (1999). Semantics in teaching introductory physics. American Journal of Physics, 67(8), 670-680. https://doi.org/10.1119/1.19351 